\title{
Stark and Kerr Effects on the Dynamics of Moving $N$-Level Atomic System
}

\author{
Syed Jamal Anwar, Mohammed Ramzan, Mohammed Usman, Mohammed Khalid Khan* \\ Department of Physics, Quaid-i-Azam University, Islamabad, Pakistan \\ Email: ^mkk@qau.edu.pk
}

How to cite this paper: Anwar, S.J., Ramzan, M., Usman, M. and Khan, M.K. (2019) Stark and Kerr Effects on the Dynamics of Moving N-Level Atomic System. Journal of Quantum Information Science, 9, 22-40. https://doi.org/10.4236/jqis.2019.91003

Received: February 5, 2019

Accepted: March 8, 2019

Published: March 11, 2019

Copyright $\odot 2019$ by author(s) and Scientific Research Publishing Inc. This work is licensed under the Creative Commons Attribution International License (CC BY 4.0).

http://creativecommons.org/licenses/by/4.0/ (c) (i) Open Access

\begin{abstract}
We have investigated numerically the dynamics of quantum Fisher information (QFI) and quantum entanglement (QE) for $N$-level atomic system interacting with a coherent field in the presence of Kerr (linear and non-linear medium) and Stark effects. It is observed that the Stark and Kerr effects play a prominent role during the time evolution of the quantum system. The evolving quantum Fisher information (QFI) is noted as time grows under the non-linear Kerr medium contrary to the $\mathrm{QE}$ for higher dimensional systems. The effect of non-linear Kerr medium is greater on the QE as we increase the value of Kerr parameter. However, QFI and QE maintain their periodic nature under atomic motion. On the other hand, linear Kerr medium has no prominent effects on the dynamics of $N$-level atomic system. Furthermore, it has been observed that QFI and QE decay soon under the influence of Stark effect. In short, the $N$-level atomic system is found prone to the change of the Kerr medium and Stark effect for higher dimensional systems.
\end{abstract}

\section{Keywords \\ QFI, Kerr Effect, Stark Effect, $N$-Level Atomic System}

\section{Introduction}

Parameter estimation is a foundation of different branches of science and technology. The newly developed methods and techniques in measurement for parameter sensitivity have led to scientific revolutions and advancement in technology. A huge work and research has been done on phase estimation related to the practical problems of state generation, loss, and decoherence [1] [2] [3]. Fisher information (FI) lies at the heart of a parameter estimation theory that was originally introduced by Fisher in 1925. Fisher information (FI) is the key concept in quantum estimation and quantum information theory [4] [5]. The in- 
formation about some system parameter can be achieved from a quantum state and thus it brings rich marks of identification for evolutions and transitions. Moreover, the FI describes the sensitivity of a state with respect to perturbation of the parameters. The larger the FI is, the higher the precision of estimation will be. The extension of the FI to the quantum regime, i.e. the quantum Fisher information (QFI), allows a characterization of this information quantity to quantum systems [6] [7], which can be explained by the quantum Cramer-Rao bound [8]. Therefore, to improve the precision, quantum resources such as coherence, entanglement, and so on are used so that the quantum states of a system can be characterized and manipulated with high precision.

Quantum entanglement $(\mathrm{QE})$ is a very famous and mysterious phenomenon of quantum mechanics that cannot be described completely. It has different applications in quantum mechanics and quantum information theory. Quantum correlations are observed in different physical systems. Quantum entanglement (QE) was first studied by Schrodinger [9] [10] as a basic phenomenon of quantum mechanics, and it had no similarity with classical approach [11]. Therefore, quantum correlations are used to calculate the quantum states of complex systems. The correlations of complex systems do not depend on the spatial separation of components, so the system behaves as a single system. Schrodinger explained that the information of different parts of the system would not contain the complete information of the whole system [11]. The quantum correlations are the results of the quantum measurements that explain the solution and information of different physical systems like the Bell inequalities [12] [13], and confirm the experimental segment of the spooky action at a distance, as presented by Einstein. In the last few years due to the progress of quantum information processing (QIP), the new field of quantum metrology becomes important and prominent [14] [15]. The importance of QE in a different process has led to the observation and investigation of larger dimensional quantum systems and shows when an important and significant role in quantum systems of many particles [16]. The real quantum systems are not closed completely and so the important quantum properties decreases. Hence, the dynamical response is observed when the system loses coherence due to interaction with the environment. When the objects are in contact with the environment, the dynamics of objects behave like an open system. Hence, the interaction between a complex quantum system and its environment and the study of the dynamics of different physical quantities are very attractive and interesting. This interaction causes a quantum noise that creates fluctuations like decoherence, and dissipative dynamics that are not reversible [17]. Therefore, in the last few decades the study of quantum decay caused by the interaction with the environment has become interesting and attractive. Moreover, the evaluation of open quantum systems has attracted attention to the study of modern quantum theory, rather more importantly to the study of quantum information processing (QIP).

The Jaynes-Cumming model (JCM) [18] represents the interaction between a 
two-level atom and cavity field of a quantized single-mode under the rotating wave approximation (RWA). It is the complicated basic model of matter--field interaction having a precise integrable Hamiltonian. The experiments done by the Rydberg atoms confirm the predictions of the JCM model [19] [20] [21] and due to this there is an improvement in the study of JCM and its different generalizations like intensity dependent JCM and multi-photon generalizations of JCM [22] [23] etc.

A Kerr-like medium is represented by a harmonic oscillator (HO) [24]. The nonlinearity of Kerr is similar to a Hamiltonian that is quadratic in the number of photon operator. The Kerr-like medium model can be presented as if the cavity is having two different types of the Rydberg atoms, one of them behaves as harmonic oscillator and the other one will couple with the field-mode. The effect of non-linear interaction of a cavity mode with a Kerr-like medium on the dynamics of atomic population has been studied when the non-linear generalization of the JCM was taken into account, [25] [26] [27]. Therefore, the interaction is mainly confined to the case of single cavity mode. Quantum dynamics of a two-level atom having interaction with two-/multi-mode cavity field in the presence of a Kerr like medium has been investigated [28] [29]. Berlin and Aliaga [30] presented the case of the non-degenerate two-photon JCM model in the presence of a Kerr-medium using a method of operators that are related to the dynamics of the system and temporal evolution is also investigated.

Recently, the JCM model was extended to consider the atomic motion along the axis of the cavity [31]. The extended standard JCM contains external atomic effects because the atomic motion is quantized and the motion of the center of the mass of an atom is cooled down to very small temperatures, hence the vibrational motion was quantized [32]. Recently properties of the atomic external and internal quantities like the radiation force and momentum of atom for the Raman-coupled JCM have been investigated [33] [34]. The interaction of a moving three level atom with two-mode cavity field including a Kerr-like medium in the corresponding cavity are investigated [35] [36]. These observations are considered when the atom is initially prepared in the eigenstate of momentum and the state of the field is squeezed. The atomic system containing a moving Rubidium atom interacting with cavity field of single mode is investigated [37]. Furthermore, the resonant case of the interaction of a moving $N$-level ladder type atom with cavity field of (N-1)-mode is observed [38]. Recently, the system having a four-level ladder type atom in a momentum eigenstate interacting with a single-mode cavity field under the influence of nonlinearities of the intensity-dependent coupling is determined [39]. Moreover, the interactions of a moving four-level $\mathrm{N}$-type atom with a three-mode cavity field in the presence of a nonlinear Kerr-like medium have been investigated [40].

The interaction of light with matter is known as Stark effect and it is a very important phenomenon in the quantum optics [41]. There is a splitting and shifting of spectral lines of atoms under the effect of electric field in this process. 
The Stark effect is investigated for the Dicke Hamiltonian in the presence of constant fields and hence shifting in eigenvalues is observed due to the emitter-cavity interaction strength. The dynamic Stark effect is observed in an optical system controlled by a laser beam. In the presence of the dipole approximation there is an exact contribution of time to the Dicke Hamiltonian. Due to the interaction with external field, it is periodic with the frequency of laser. The shift in Rabi splitting is observed due to dynamic Stark effect. The recent progress in the field of cavity quantum electrodynamics (QED) [42] [43] made it possible to achieve the strong and ultra-strong light-matter-interaction patterns experimentally. Therefore, the full Dicke Hamiltonian drawing on the outer laser is controlled to represent the dynamical properties The Stark shift explains the multi-photon transition in more precise and accurate manner. The significance of this process can be noticeable, when two levels of atom interact with the middle level [44]. The Stark shift mechanism is explained in this situation by considering the process of adiabatic elimination of the middle level(s) of a multi-level atom [45]. This method calculates a simple effective Hamiltonian of a two-level atom interacting with a quantized field of single-mode with at least two-photon transition in the presence of Stark shift [46]. The Stark shift in two-photon transitions is taken as the detuning depending on the intensity [47]. The interaction of two two-level atoms and a field of single-mode with degenerate two-photon transition in the presence of the Stark shift are investigated [48] and it is described that the degree of QE increases with the rise in the value of the Stark shift parameter.

von Neumann entropy (VNE) is considered the main entanglement measure for pure states. The correlation between quantum entanglement and Fisher information (FI) about a certain parameter in a quantum state has not been studied widely. Moreover, observations have been made to calculate the QE of pure state by using FI. In this regard, the entanglement evaluation and QFI of $N$-level atomic system under the influence of intrinsic decoherence is studied [49]. Also, the time evolution of the QFI of a system whose dynamics is described by the phase-damped model has been studied [50]. It is observed that there is an interesting monotonic relation between the QFI and nonlocal correlation behavior measured by the negativity depending on choosing the estimator parameter during the time evolution.

In this present paper our main focus is to investigate the $\mathrm{QE}$ and dynamics $N$-level moving atomic system which is interacting with coherent field in presence of Kerr medium and Stark shift. We have calculated VNE and QFI of the $N$-level atom system in the presence of atomic motion and without atomic motion.

The paper is arranged as follows. In Section 2, we present the background related to VNE and QFI. The system Hamiltonian and dynamics are presented in Section 3 for $N$-level atomic system affected by Kerr and Stark effect. Section 4 provides detailed results and numerical discussions. In Section 5, we present a 
brief conclusion.

\section{Introduction to Quantum Fisher Information (QFI)}

The classical Fisher information (CFI) for any particular phenomenon with a one parameter $\theta$ which is unknown can be written as

$$
I_{\Phi}=\sum_{i} p_{i}(\Phi)\left[\frac{\partial}{\partial \Phi} \ln p_{i}(\Phi)\right]^{2},
$$

where $p_{i}(\Phi)$ is representing the probability density having significant and prominent influence on the fixed parameter with the outcome $\left\{x_{i}\right\}$ of measurement for a distinct observable $X$. The CFI can be described with the help of the inverse variance of the asymptotic normality of a maximum-likelihood estimator. The QFI has a very important and effective part in quantum metrology and the large precise measurement of an unknown parameter can be gained which is having relation to inverse of the quantum fisher information and is described as [51].

$$
F_{\Phi}=\operatorname{Tr}\left[\rho(\theta) D^{2}\right],
$$

where $\rho(\Phi)$ is describing the density matrix (DM) of the system, $\Phi$ is the parameter which will be estimated, and $L$ is representing the quantum score (symmetric logarithmic derivative) which is written as

$$
\frac{\mathrm{d} \rho(\Phi)}{\mathrm{d} \Phi}=\frac{1}{2}[\rho(\Phi) D+D \rho(\Phi)] \text {. }
$$

here we are considering $N$-level atomic system with the density operator $\rho(\Phi)$. The spectral decomposition of the DM is described as

$$
\rho_{\Phi}=\sum_{K} \lambda_{K}|k\rangle\langle k|,
$$

The QFI which is related to $\Phi$ for this DM is represented by [52]

$$
F_{\Phi}=\sum_{k} \frac{\left(\partial_{\Phi} \lambda_{k}\right)^{2}}{\lambda_{k}}+2 \sum_{k, k^{\prime}} \frac{\left(\lambda_{k}-\lambda_{k^{\prime}}\right)^{2}}{\left(\lambda_{k}+\lambda_{k^{\prime}}\right)}\left|\left\langle k \mid \partial_{\Phi} k^{\prime}\right\rangle\right|^{2}
$$

where $\lambda_{k}>0$ and $\lambda_{k}+\lambda_{k^{\prime}}>0$. The first term in above equation is representing CFI and the second term describes QFI. The AQFI is calculated by taking the trace over the field. Therefore, we will be able to represent the AQFI of a bipartite density operator $\rho_{A B}$ which is related to $\Phi$ as [53]

$$
I_{Q F}(t)=I_{A B}(\Phi, t)=\operatorname{Tr}\left[\rho_{A B}(\Phi, t)\{D(\Phi, t)\}^{2}\right]
$$

where $D(\Phi, t)$ is representing the quantum score [54] (the symmetric logarithmic derivative) that will be written as

$$
\frac{\partial \rho_{A B}(\Phi, t)}{\partial \Phi}=\frac{1}{2}\left[D(\Phi, t) \rho_{A B}(\Phi, t)+\rho_{A B}(\Phi, t) D(\Phi, t)\right]
$$

In the same way we will define the von Neumann entropy as

$$
S_{A}=-\operatorname{Tr}\left(\rho_{A} \ln \rho_{A}\right)=-\sum_{i} r_{i} \ln r_{i}
$$


where $r_{i}$ represents the eigenvalues of the atomic DM $\rho_{A}=\operatorname{Tr}_{B}\left(\rho_{A B}\right)$.

\section{Model and Hamiltonian}

We consider the model consisting of moving $N$-level atoms interacting with a single-mode cavity field. The total Hamiltonian of the system $\hat{H}_{T}$ under the RWA for atom-field system can be described as [55]

$$
\hat{H}_{T}=\hat{H}_{\text {Atom-Field }}+\hat{H}_{I} \text {. }
$$

where $\hat{H}_{\text {Atom-Field }}$ is representing the Hamiltonian for the non-interacting atom and field, and the interaction part is given by $\hat{H}_{I}$. We will write $\hat{H}_{\text {Atom-Field }}$ as

$$
\hat{H}_{\text {Atom-Field }}=\sum_{j} \omega_{j} \hat{\sigma}_{j, j}+\Omega \hat{a}^{\dagger} \hat{a},
$$

where $\hat{\sigma}_{j, j}=|j\rangle\langle j|$ are describing as population operators for the jth level. The interaction Hamiltonian of $\mathrm{N}$-level atomic system for the case that is not resonant is written as [56]

$$
\hat{H}_{I}=\sum_{s=1}^{N} \Omega(t)\left[\hat{a} \mathrm{e}^{-i \Delta_{s} t} \hat{\sigma}_{s, s+1}+\left(\hat{a} \mathrm{e}^{-i \Delta_{s} t} \hat{\sigma}_{s, s+1}\right)^{\dagger}\right] .
$$

In the case of Kerr-like medium, the interaction Hamiltonian can be written as

$$
\hat{H}_{I}=\sum_{s=1}^{N} \Omega(t)\left[\hat{a} \mathrm{e}^{-i \Delta_{s} t} \hat{\sigma}_{s, s+1}+\left(\hat{a} \mathrm{e}^{-i \Delta_{s} t} \hat{\sigma}_{s, s+1}\right)^{\dagger}\right]+\chi\left(\hat{a}^{\dagger} \hat{a}\right)^{q},
$$

and in case of linear Kerr-like medium $q=1$ and in case of non-linear Kerr medium $q=2$, so the interaction Hamiltonian for linear and non-linear Kerr medium is given as

$$
\begin{aligned}
& \hat{H}_{I}=\sum_{s=1}^{N} \Omega(t)\left[\hat{a} \mathrm{e}^{-i \Delta_{s} t} \hat{\sigma}_{s, s+1}+\left(\hat{a} \mathrm{e}^{-i \Delta_{s} t} \hat{\sigma}_{s, s+1}\right)^{\dagger}\right]+\chi\left(\hat{a}^{\dagger} \hat{a}\right)^{1}, \\
& \hat{H}_{I}=\sum_{s=1}^{N} \Omega(t)\left[\hat{a} \mathrm{e}^{-i \Delta_{s} t} \hat{\sigma}_{s, s+1}+\left(\hat{a} \mathrm{e}^{-i \Delta_{s} t} \hat{\sigma}_{s, s+1}\right)^{\dagger}\right]+\chi\left(\hat{a}^{\dagger} \hat{a}\right)^{2},
\end{aligned}
$$

and when the Stark effect is included in the interaction Hamiltonian, it can be written as

$$
\hat{H}_{I}=\sum_{s=1}^{N} \Omega(t)\left[\hat{a} \mathrm{e}^{-i \Delta_{s} t} \hat{\sigma}_{s, s+1}+\left(\hat{a} \mathrm{e}^{-i \Delta_{s} t} \hat{\sigma}_{s, s+1}\right)^{\dagger}\right]+\beta \hat{a}^{\dagger} \hat{a}|g\rangle\langle g| .
$$

where $g$ is representing the ground state of $N$-level atomic system and $\chi$ and $\beta$ are the constants of Kerr-like medium and Stark effect.

We will describe the detuning parameter as

$$
\Delta_{s}=\Omega+\omega_{s+1}-\omega_{s},
$$

and the coupling constant for atom and field is $G, \Omega(t)$ is representing the shape function of the cavity-field mode [57] and the motion of atom is along $z$-axis. For particular interest

$$
\begin{aligned}
& \Omega(t)=G \sin (w \pi v t / L) \text { in the presence of atomic motion, } w \neq 0 \\
& \Omega(t)=G \text { in the absence of atomic motion } w=0
\end{aligned}
$$


where the velocity of motion of atom is $v$ and $w$ denotes the wavelengths of half the number of the mode in the cavity and $L$ describes the length of cavity along $z$ direction. Now take the velocity of atom as $v=\lambda L / \pi$ which gives us

$$
\begin{aligned}
\Omega_{1}(t)=\int_{0}^{t} \Omega(\tau) \mathrm{d} \tau= & \frac{1}{w}(1-\cos (w \pi v t / L) \text { for } w \neq 0 \\
& =G t \text { for } w=0 .
\end{aligned}
$$

In order to find the phase shift parameter as precisely as possible, we consider the optimal input state as

$$
|\Psi(0)\rangle_{\mathrm{opt}}=\frac{1}{\sqrt{2}}(|1\rangle+|0\rangle) \otimes|\alpha\rangle
$$

where $|1\rangle$ and $|0\rangle$ describe the states of atom and $\alpha$ is the coherent state of the input field given as

$$
|\alpha\rangle=\sum_{n=0}^{\infty} \alpha^{n} \sqrt{\mathrm{e}^{-|\alpha|^{2}} / n !}|n\rangle .
$$

In order to introduce the phase shift parameter $\phi$, we consider a single-atom phase gate that introduces the phase shift as

$$
\hat{U}_{\phi}=|1\rangle\left\langle 1\left|+\mathrm{e}^{i \phi}\right| 0\right\rangle\langle 0|,
$$

$|\Psi(0)\rangle$ is obtained from the operation of the single-atom phase gate on $|\Psi(0)\rangle_{\mathrm{opt}}$

$$
\begin{aligned}
& \hat{U}_{\phi}|\Psi(0)\rangle_{\mathrm{Opt}}=|\Psi(0)\rangle \\
& =\frac{1}{\sqrt{2}}\left(|1\rangle+\mathrm{e}^{i \phi}|0\rangle\right) \otimes|\alpha\rangle
\end{aligned}
$$

After the operation of phase gate, the system will interact with a field. The precision of the estimation is strongly affected by the characteristics of the interaction between the field and moving $N$-level atomic system.

Now we can write the state $|\Psi(0)\rangle$ as

$$
|\Psi(0)\rangle=\frac{1}{\sqrt{2}}\left(|1, n+1\rangle+\mathrm{e}^{i \phi}|0, n\rangle\right)
$$

where $|1, n+1\rangle$ and $|0, n\rangle$ are allowable atom-field states. The states in which a number of photons are consistent with the atomic level are known as allowable atom field states. For our $N$-level atomic system, the allowable basis are given by

$$
|0, n\rangle,|1, n+1\rangle,|2, n+1\rangle, \cdots,|N-2,(n+N-2)\rangle,|N-1,(n+N-1)\rangle,
$$

where $n$ are number of photons initially present in the cavity and $N-1$ are the number of levels in an atom. 0 and $N-1$ represents the excited and ground state of the atom.

Let us consider the time-independent case, characterized by the transformation matrix $\hat{U}(t)$. The wave function

$$
|\Psi(t)\rangle=\hat{U}(t)|\Psi(0)\rangle,
$$

In the next section, the influence of the parameters $w, \phi, \chi$ and $\beta$ on the 
evolution of the $A Q F I$ and $V N E$ is presented.

\section{Discussions of Numerical Results}

In this section we will present the results of the time evolution of QFI and von Neumann entropy of a system of $N$-level atom interacting with a coherent field under the influence of Stark and Kerr effects. For the sake of simplicity, we scaled out the time $t$ i.e. one unit of time is described by the inverse of the coupling constant $G$. Initially, we investigate the time evolution of QFI and Von Neumann entropy for $N$-level atomic system interacting with a non linear Kerr medium with and without atomic motion. In Figure 1, we plot the QFI and von Neumann entropy as a function of time for $N$-level atoms interacting with coherent field under the influence of a non linear Kerr medium for $|\alpha|^{2}=6, \quad \chi=1$, phase shift $\phi=0, \pi / 4$ and atomic motion parameter $w=0$. It is seen that QFI evolves as time grows contrary to the entanglement for higher dimensional systems.

In Figure 2, we plot the QFI and von Neumann entropy as a function of time for the system of $N$-level atom having interaction with coherent field for $|\alpha|^{2}=6, \chi=3$, phase shift $\phi=0, \pi / 4$ without atomic motion i.e. $w=0$. It has been observed that the effect of non-linear Kerr medium is immense on the entanglement as we increase the Kerr effect. These results show the strong dependence of QFI and entanglement on the Kerr-like medium.

In Figure 3 and Figure 4 we plot QFI and von Neumann entropy as a function of time for $N$-level atom interacting with coherent field for $|\alpha|^{2}=6$, $\chi=1,3$ respectively along with phase shift $\phi=0, \pi / 4$ and atomic motion parameter $w=1$ A sudden increase of entanglement is observed for the five-level atomic system at $\chi=3$. Five level-Atom has more probability to interact with field as compare to three and four-level atom at larger value of non-linear Kerr parameter $\chi=3$, as the unitary operator is written as superposition of two terms and its difference from five-level atom is more significant as compared to three and four-level atom, so it means that the higher dimensional atom like five level atom interact more with field at larger values of Kerr-like medium. Where as a periodic behaviour of QFI and entanglement is observed in the presence of atomic motion.

In Figures 5-8 we plot QFI and von Neumann entropy as a function of time for $N$-level atomic system interacting with coherent field for $|\alpha|^{2}=6$, with linear Kerr-like medium $\chi=1,3$, phase shift parameter $\phi=0, \pi / 4$ with and without atomic motion i.e. $w=0$ and 1. It is seen that the linear Kerr medium has no prominent effect on the dynamics of $N$-level system as compared to the effect of non-linear Kerr medium.

In Figures 9-12 we plot QFI and von Neumann entropy as a function of time for $N$-level atomic system interacting with coherent field for $|\alpha|^{2}=6, \chi=1,3$, phase shift parameter $\phi=0, \pi / 4, \quad \beta=1,3$ and atomic motion parameter $w=0$ and 1 respectively. It is seen that QFI and entanglement decay promptly 

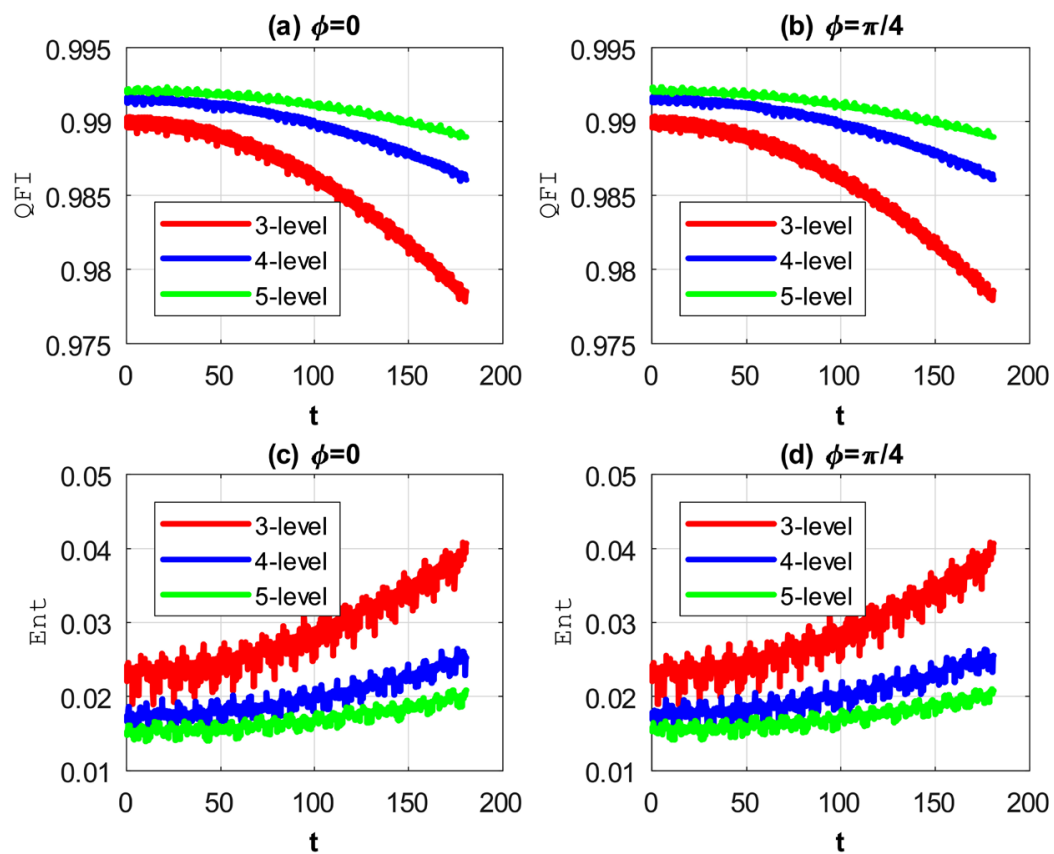

Figure 1. (Color online) The QFI (upper panel) and von Neumann entropy (lower panel) as a function of time for a system of $N$-level atom having interaction with coherent field for $|\alpha|^{2}=6$ and the phase shift estimator parameter $\phi=0$ (left panel) and $\pi / 4$ (right panel). The parameter $w$ of atomic motion is ignored and value of $\chi=1$ (Non-linear kerr).
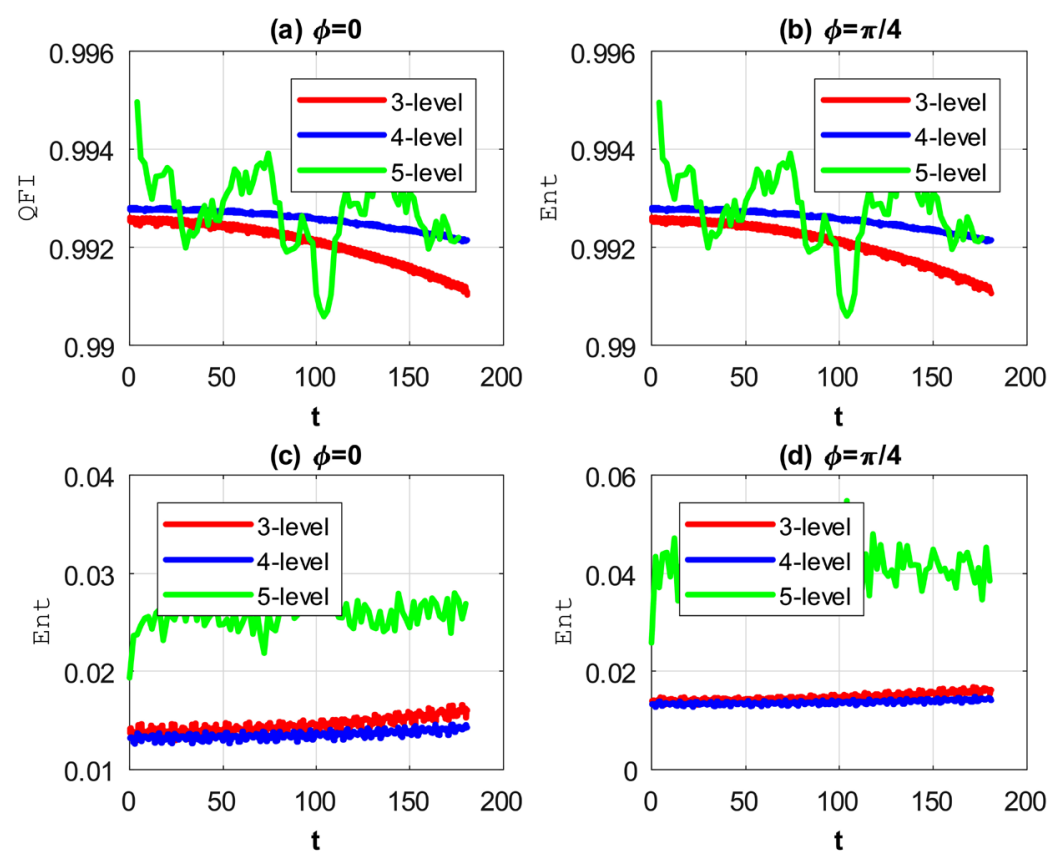

Figure 2. (Color online) The QFI (upper panel) and von Neumann entropy (lower panel) as a function of time for a system of $N$-level atom having interaction with coherent field for $|\alpha|^{2}=6$ and the phase shift estimator parameter $\phi=0$ (left panel) and $\pi / 4$ (right panel). The parameter $w$ of atomic motion is ignored and value of $\chi=3$ (Non-linear kerr). 

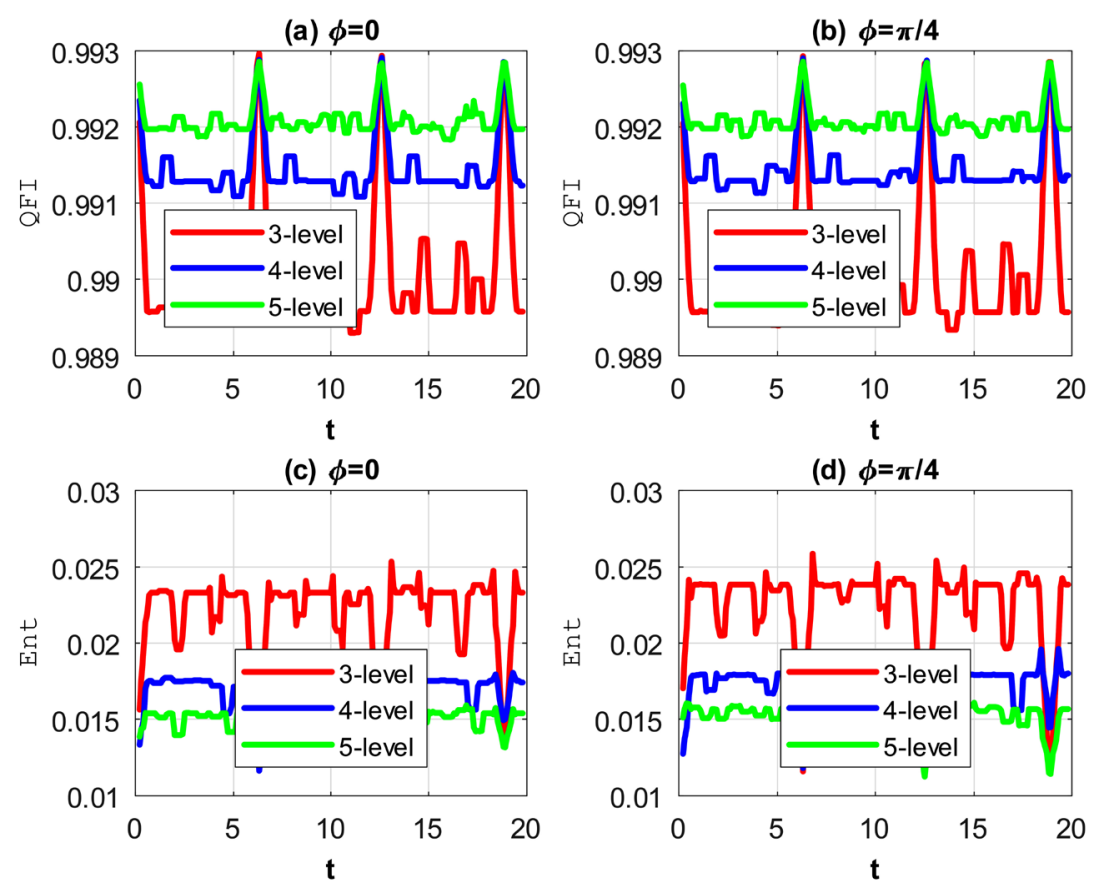

Figure 3. (Color online) The QFI (upper panel) and von Neumann entropy (lower panel) as a function of time for a system of $N$-level atom having interaction with coherent field for $|\alpha|^{2}=6$ and the phase shift estimator parameter $\phi=0$ (left panel) and $\pi / 4$ (right panel). The parameter $w$ of atomic motion is 1 and value of $\chi=1$ (Non-linear kerr).

(a) $\phi=0$

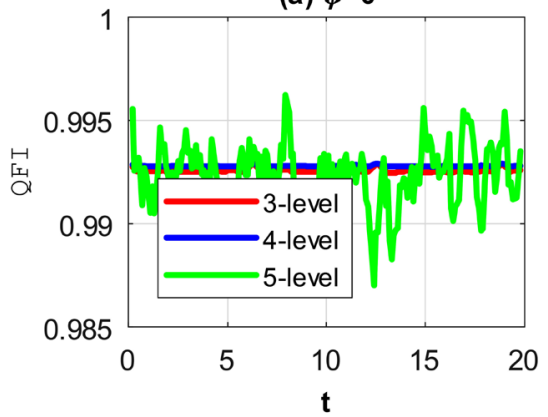

(c) $\phi=0$

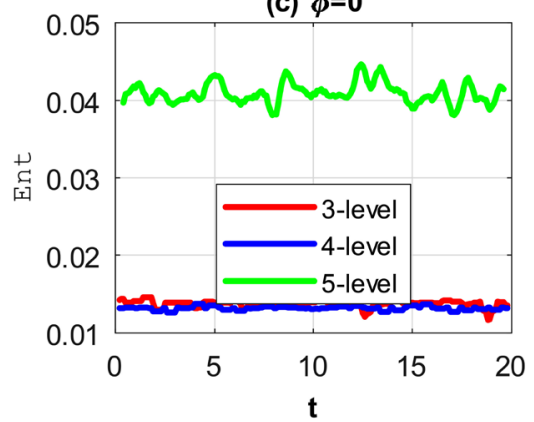

(b) $\phi=\pi / 4$

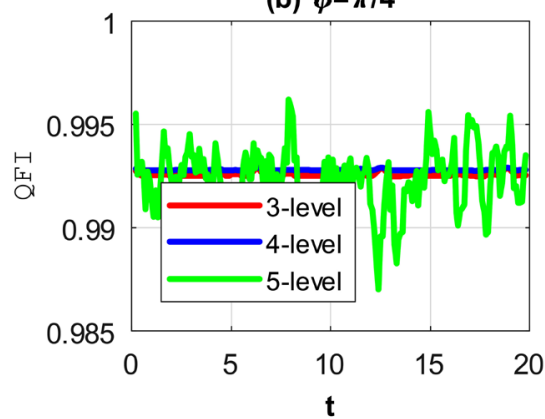

(d) $\phi=\pi / 4$

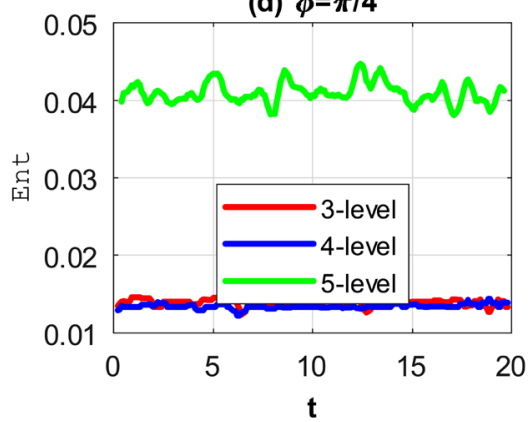

Figure 4. (Color online) The QFI (upper panel) and von Neumann entropy (lower panel) as a function of time for a system of $\mathrm{N}$-level atom having interaction with coherent field for $|\alpha|^{2}=6$ and the phase shift estimator parameter $\phi=0$ (left panel) and $\pi / 4$ (right panel). The parameter $w$ of atomic motion is 1 and value of $\chi=3$ (Non-linear kerr). 
(a) $\phi=0$

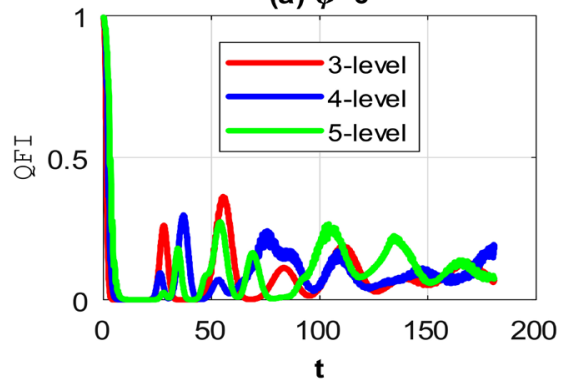

(c) $\phi=0$

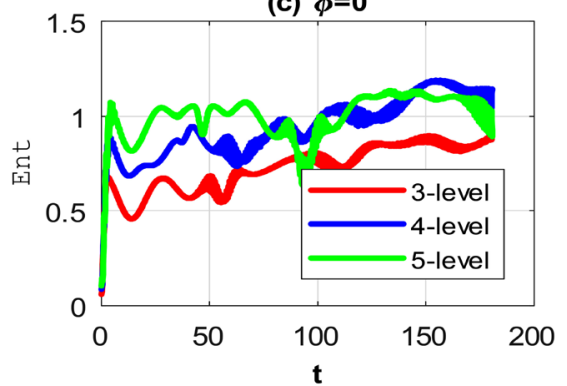

(b) $\phi=\pi / 4$

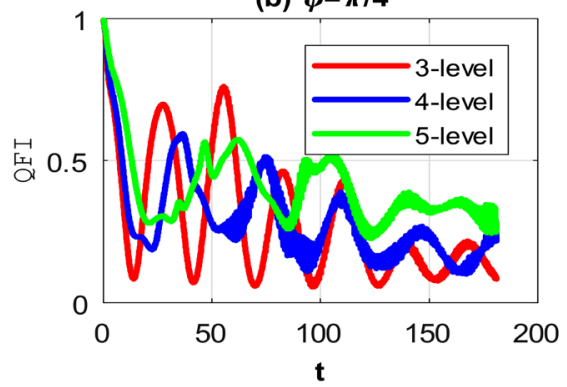

(d) $\phi=\pi / 4$

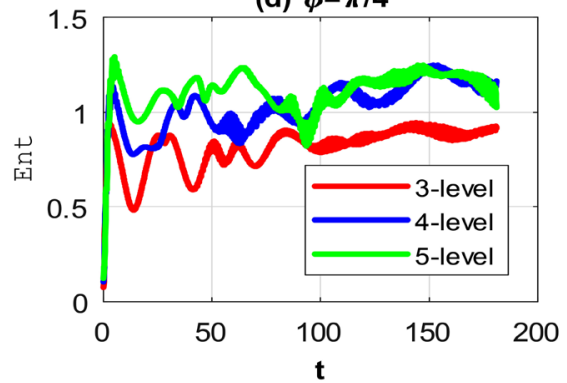

Figure 5. (Color online) The QFI (upper panel) and von Neumann entropy (lower panel) as a function of time for a system of $N$-level atom having interaction with coherent field for $|\alpha|^{2}=6$ and the phase shift estimator parameter $\phi=0$ (left panel) and $\pi / 4$ (right panel). The parameter $w$ of atomic motion is ignored and value of $\chi=1$ (Linear kerr).

(a) $\phi=0$

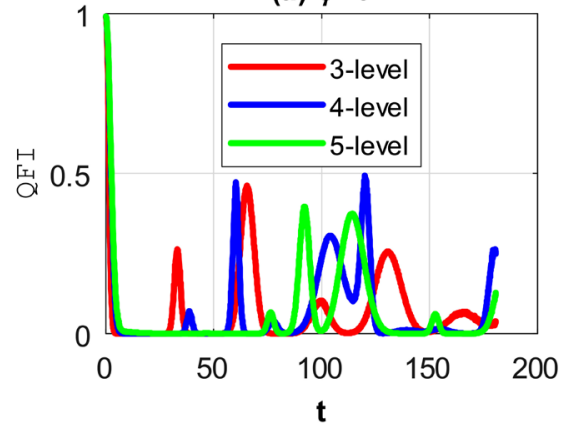

(c) $\phi=0$

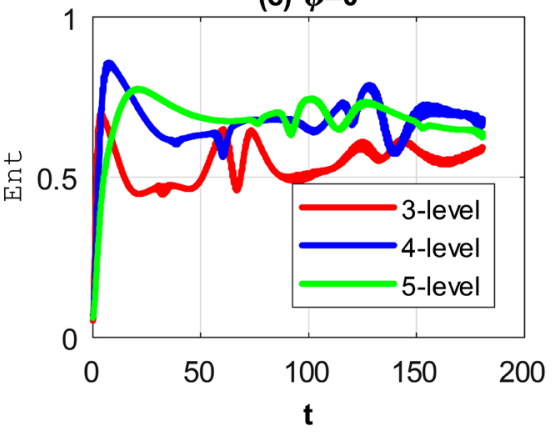

(b) $\phi=\pi / 4$

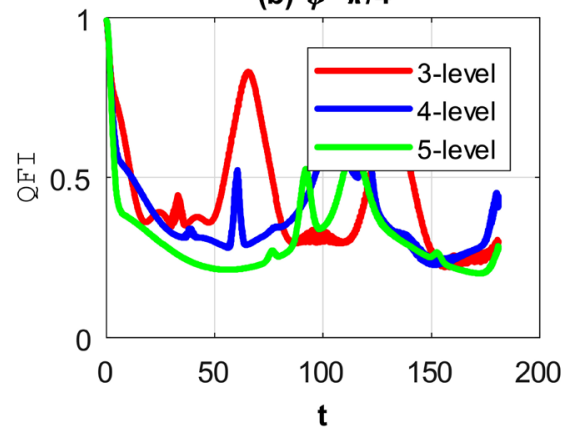

(d) $\phi=\pi / 4$

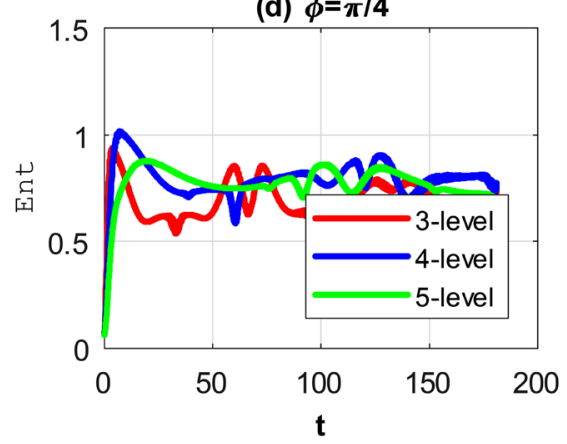

Figure 6. (Color online) The QFI (upper panel) and von Neumann entropy (lower panel) as a function of time for a system of $N$-level atom having interaction with coherent field for $|\alpha|^{2}=6$ and the phase shift estimator parameter $\phi=0$ (left panel) and $\pi / 4$ (right panel). The parameter $w$ of atomic motion is ignored and value of $\chi=3$ (Linear kerr). 
(a) $\phi=0$

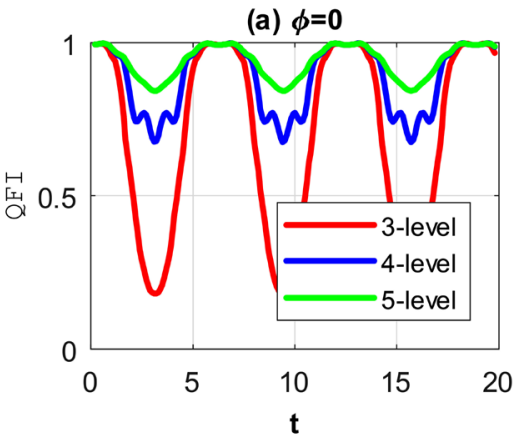

(c) $\phi=0$

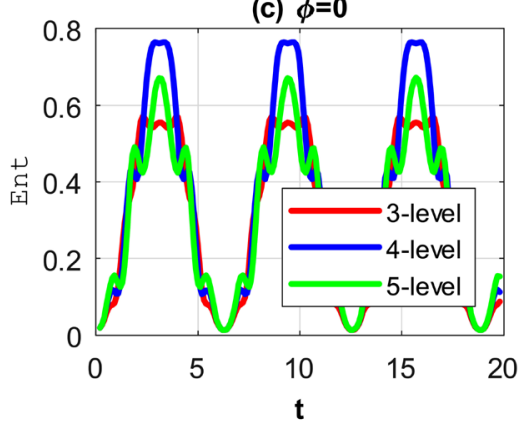

(b) $\phi=\pi / 4$

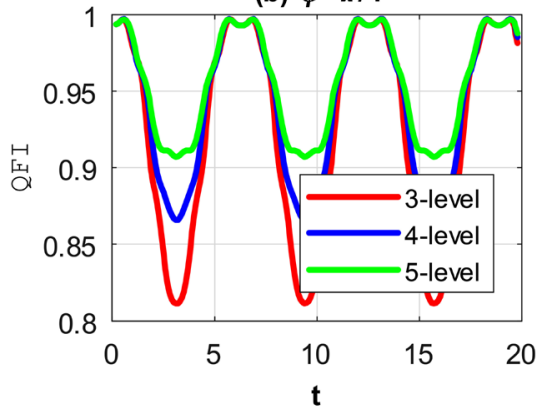

(d) $\phi=\pi / 4$

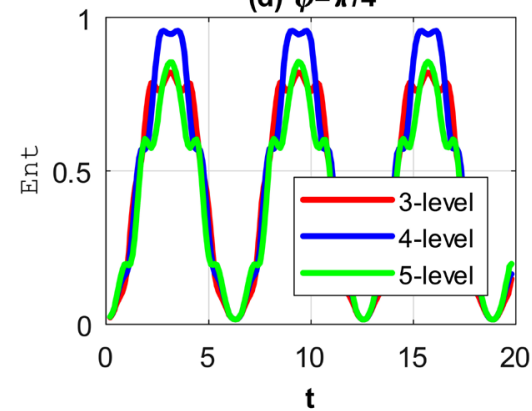

Figure 7. (Color online) The QFI (upper panel) and von Neumann entropy (lower panel) as a function of time for a system of $N$-level atom having interaction with coherent field for $|\alpha|^{2}=6$ and the phase shift estimator parameter $\phi=0$ (left panel) and $\pi / 4$ (right panel). The parameter $w$ of atomic motion is 1 and value of $\chi=1$ (Linear kerr).

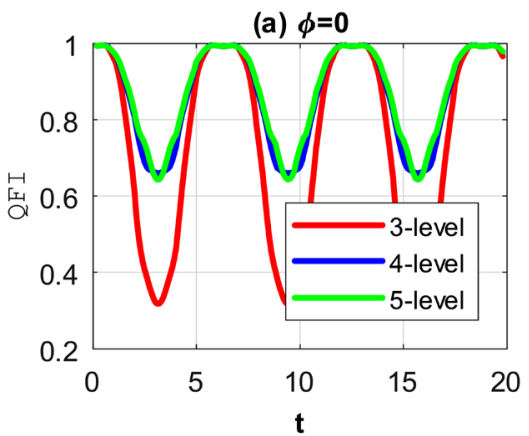

(c) $\phi=0$

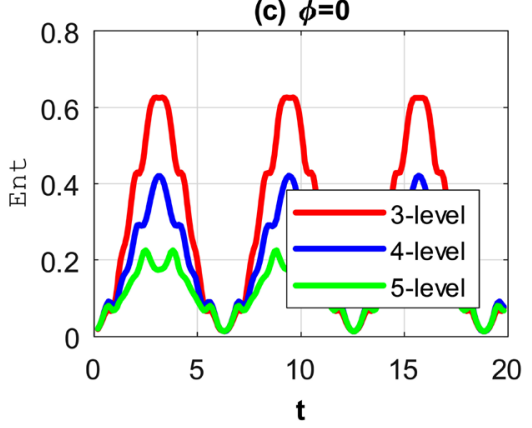

(b) $\phi=\pi / 4$

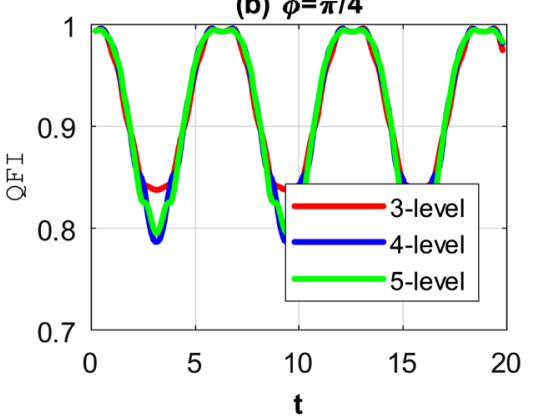

(d) $\phi=\pi / 4$

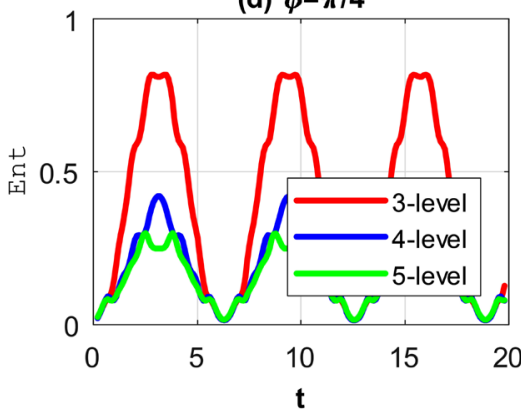

Figure 8. (Color online) The QFI (upper panel) and von Neumann entropy (lower panel) as a function of time for a system of $\mathrm{N}$-level atom having interaction with coherent field for $|\alpha|^{2}=6$ and the phase shift estimator parameter $\phi=0$ (left panel) and $\pi / 4$ (right panel). The parameter $w$ of atomic motion is 1 and value of $\chi=3$ (Linear kerr). 
(a) $\phi=0$

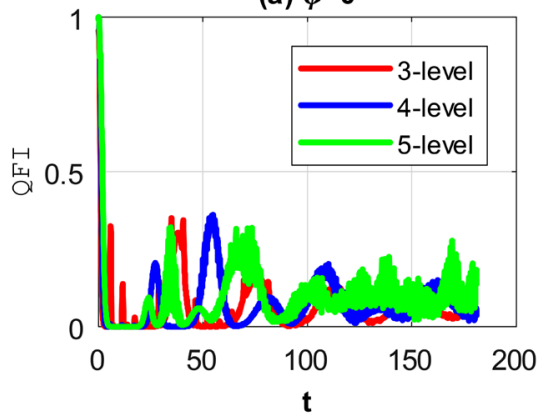

(c) $\phi=0$

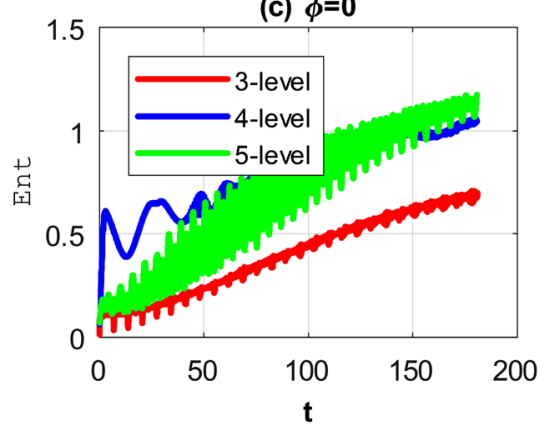

(b) $\phi=\pi / 4$

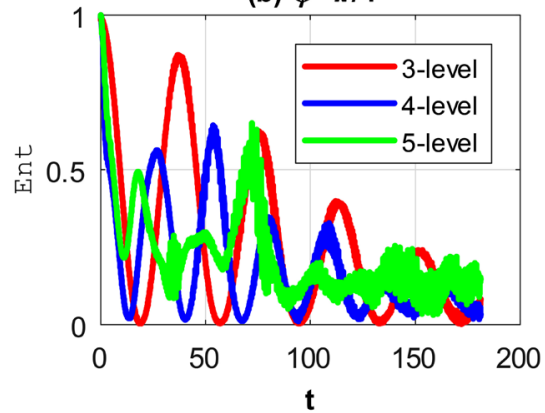

(d) $\phi=\pi / 4$

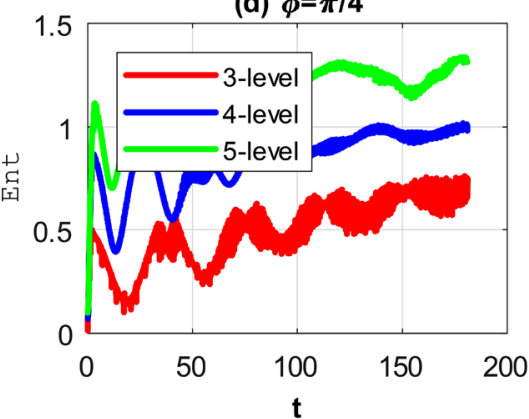

Figure 9. (Color online) The QFI (upper panel) and von Neumann entropy (lower panel) as a function of time for a system of $N$-level atom having interaction with coherent field for $|\alpha|^{2}=6$ and the phase shift estimator parameter $\phi=0$ (left panel) and $\pi / 4$ (right panel). The parameter $w$ of atomic motion is ignored and value of $\beta=1$ (Stark effect).

(a) $\phi=0$

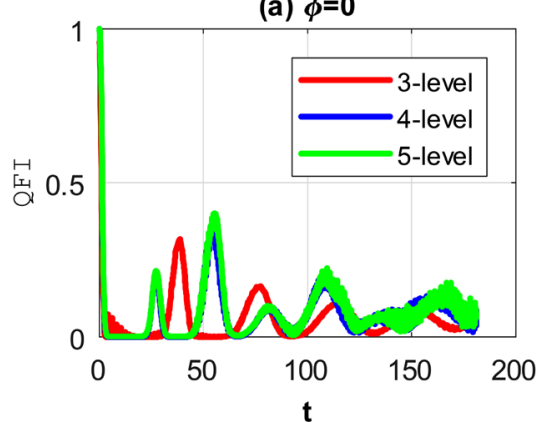

(c) $\phi=0$

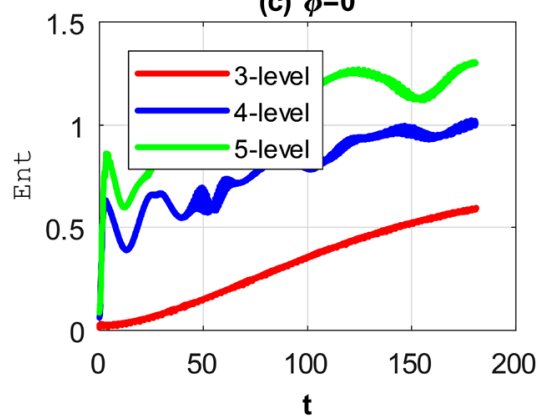

(b) $\phi=\pi / 4$

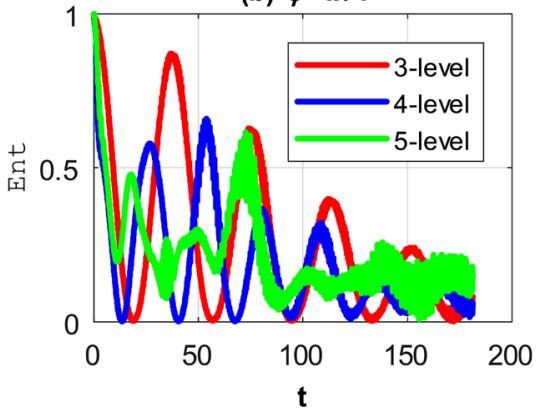

(d) $\phi=\pi / 4$

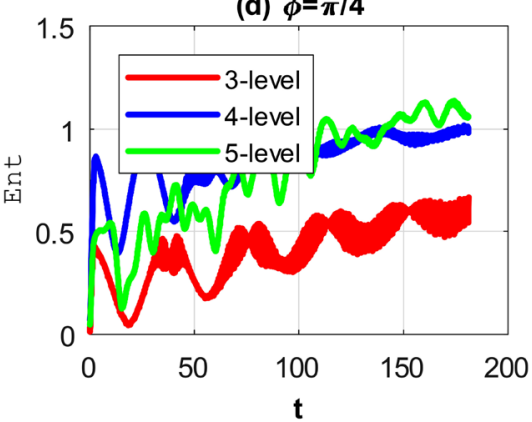

Figure 10. (Color online) The QFI (upper panel) and von Neumann entropy (lower panel) as a function of time for a system of $N$-level atom having interaction with coherent field for $|\alpha|^{2}=6$ and the phase shift estimator parameter $\phi=0$ (left panel) and $\pi / 4$ (right panel). The parameter $w$ of atomic motion is ignored and value of $\beta=3$ (Stark effect). 
(a) $\phi=0$

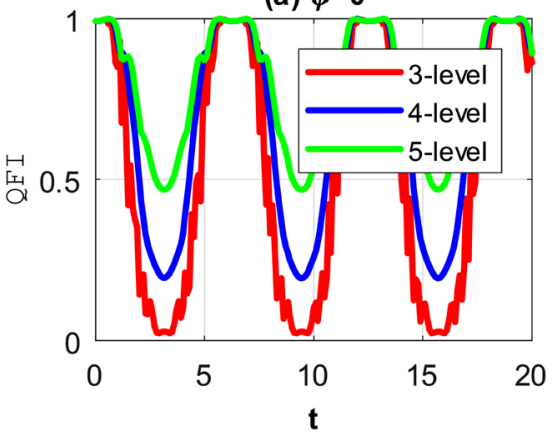

(c) $\phi=0$

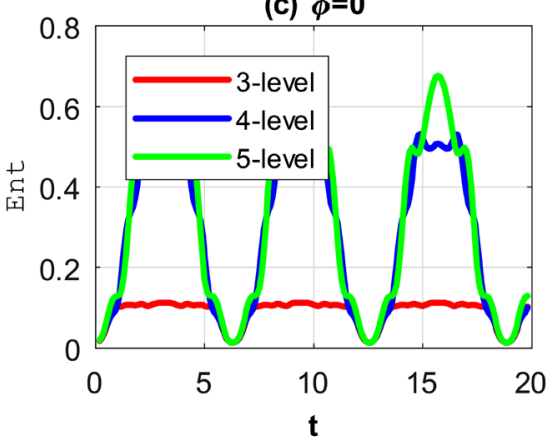

(b) $\phi=\pi / 4$

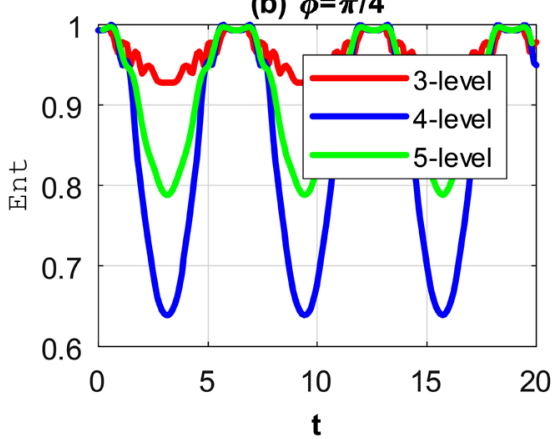

(d) $\phi=\pi / 4$

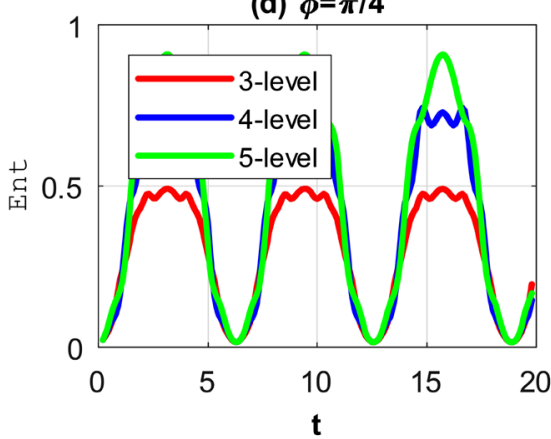

Figure 11. (Color online) The QFI (upper panel) and von Neumann entropy (lower panel) as a function of time for a system of $N$-level atom having interaction with coherent field for $|\alpha|^{2}=6$ and the phase shift estimator parameter $\phi=0$ (left panel) and $\pi / 4$ (right panel). The parameter $w$ of atomic motion is 1 and value of $\beta=1$ (Stark effect).

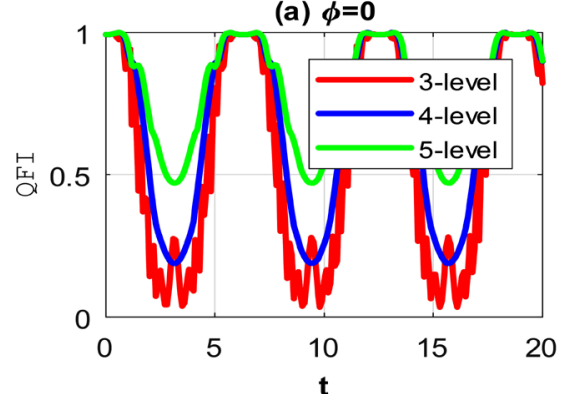

(c) $\phi=0$

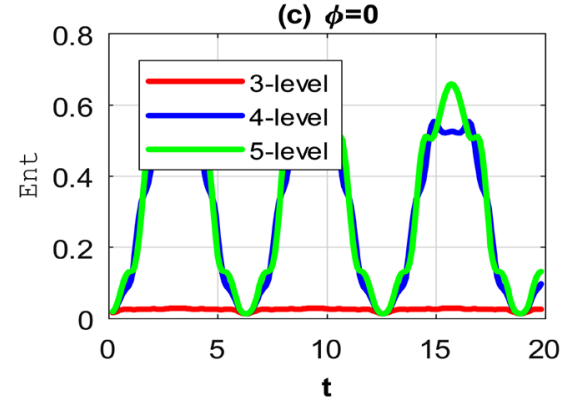

(b) $\phi=\pi / 4$

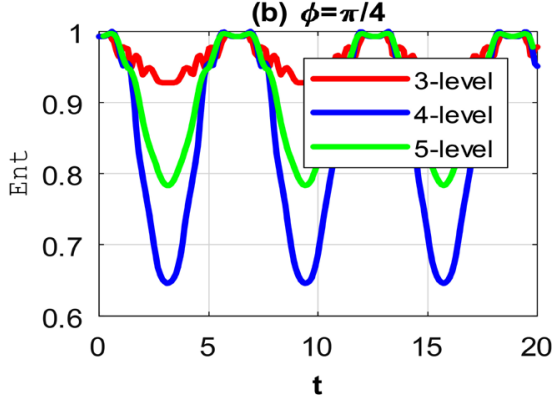

(d) $\phi=\pi / 4$

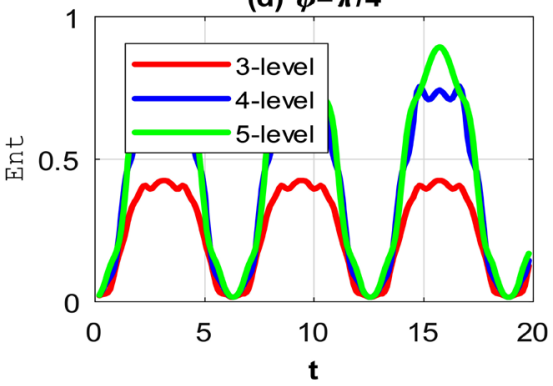

Figure 12. (Color online) The QFI (upper panel) and von Neumann entropy (lower panel) as a function of time for a system of $N$-level atom having interaction with coherent field for $|\alpha|^{2}=6$ and the phase shift estimator parameter $\phi=0$ (left panel) and $\pi / 4$ (right panel). The parameter $w$ of atomic motion is 1 and value of $\beta=3$ (Stark effect). 
under the influence of Stark effect. Finally, we conclude that the $N$-level atomic system is fully prone to the change of the Kerr-like medium and Stark effect at higher dimensions.

\section{Conclusion}

We study the dynamical evolution of QFI and entanglement for $N$-level atomic system in the presence of Kerr (linear and non-linear medium) and Stark effects. The time evolution of the atomic system interacting with a coherent field under the influence of Stark and kerr effect is investigated numerically. It is shown that Stark and Kerr effect play a significant role during the time evolution of the quantum system. It is seen that QFI evolves as time grows contrary to the entanglement for higher dimensional systems under the influence of non-linear Kerr medium. However, the effect of non-linear Kerr medium is immense on the entanglement for larger values of Kerr medium. A sudden jump of entanglement is observed for $N$-level system (five-level atom) at $\chi=3$ because at larger values of non-linear Kerr parameter $\chi$, five-level atom has more probability to interact with field as compared to three- and four-level atom. On the one hand, a periodic behaviour of QFI and entanglement is observed in the presence of atomic motion. On the other hand, linear Kerr medium has no prominent effects on the dynamics of $N$-level system. Furthermore, QFI and entanglement decay promptly under the influence of Stark effect. Finally, we conclude that the $\mathrm{N}$-level atomic system is found fully prone to the change of the Kerr medium and Stark effect for higher-level atomic systems.

\section{Conflicts of Interest}

The authors declare no conflicts of interest regarding the publication of this paper.

\section{References}

[1] Giovannetti, V., Lloyd, S. and Maccone, L. (2004) Quantum-Enhanced Measurements: Beating the Standard Quantum Limit. Science, 306, 1330-1336. https://doi.org/10.1126/science.1104149

[2] Dowling, J. (2008) Quantum Optical Metrology-The Lowdown on High-N00N States. Contemporary Physics, 49, 125-143. https://doi.org/10.1080/00107510802091298

[3] Higgins, B.L., Berry, D.W., Bartlett, S.D., Wiseman, H.M. and Pryde, G.J. (2007) Entanglement-Free Heisenberg-Limited Phase Estimation. Nature, 450, 393-396. https://doi.org/10.1038/nature06257

[4] Helstrom, C.W. (1976) Quantum Detection and Estimation Theory. Academic Press, New York.

[5] Holevo, A.S. (1982) Statistical Structure of Quantum Theory. North-Holland, Amsterdam.

[6] Braunstein, S.L. and Caves, C.M. (1994) Statistical Distance and the Geometry of Quantum States. Physical Review Letters, 72, 3439.

https://doi.org/10.1103/PhysRevLett.72.3439 
[7] Braunstein, S.L., Caves, C.M. and Milburn, G.J. (1996) Generalized Uncertainty Relations: Theory, Examples, and Lorentz Invariance. Annals of Physics, 247, 135-173. https://doi.org/10.1006/aphy.1996.0040

[8] Hubner, M. (1992) Explicit Computation of the Bures Distance for Density Matrices. Physics Letters A, 163, 239-242. https://doi.org/10.1016/0375-9601(92)91004-B

[9] Einstein, A., Podolsky, B. and Rosen, N. (1935) Can Quantum-Mechanical Description of Physical Reality Be Considered Complete? Physical Review, 47, 777. https://doi.org/10.1103/PhysRev.47.777

[10] Schrdinger, E. (1935) Discussion of Probability Relations between Separated Systems. Mathematical Proceedings of the Cambridge Philosophical Society, 31, 555-563. https://doi.org/10.1017/S0305004100013554

[11] Nielsen, M.A. and Chuang, I.L. (2000) Quantum Computation and Information. Cambridge University Press, Cambridge.

[12] Bell, J. (1964) On the Einstein Podolsky Rosen paradox. Physics, 1, 195. https://doi.org/10.1103/PhysicsPhysiqueFizika.1.195

[13] Clauser, J., Horne, M., Shimony, A. and Holt, R. (1969) Proposed Experiment to Test Local Hidden-Variable Theories. Physical Review Letters, 23, 880. https://doi.org/10.1103/PhysRevLett.23.880

[14] Huver, S.D., Wildfeuer, C.F. and Dowling, J.P. (2008) Entangled Fock States for Robust Quantum Optical Metrology, Imaging, and Sensing. Physical Review A, 78, Article ID: 063828. https://doi.org/10.1103/PhysRevA.78.063828

[15] Berrada, K. (2013) Quantum Metrology with SU(1,1) Coherent States in the Presence of Nonlinear Phase Shifts. Physical Review A, 88, Article ID: 013817. https://doi.org/10.1103/PhysRevA.88.013817

[16] Amico, L., Fazio, R., Osterloh, A. and Vedral, V. (2008) Entanglement in Many-Body Systems. Reviews of Modern Physics, 80, 517-576. https://doi.org/10.1103/RevModPhys.80.517

[17] Nieuwenhuizen, T.M., Pomb, C., Furtado, C., et al. (2014) Quantum Foundations and Open Quantum Systems. World Scientific, Singapore. https://doi.org/10.1142/9238

[18] Jaynes, E.T. and Cummings, F.W. (1963) Comparison of Quantum and Semiclassical Radiation Theories with Application to the Beam Maser. Proceedings of the IEEE, 51, 89-109. https://doi.org/10.1109/PROC.1963.1664

[19] Meschede, D. (1992) Cavity Quantum Electrodynamics. Reports on Progress in Physics, 211, 201-250. https://doi.org/10.1016/0370-1573(92)90110-L

[20] Hansch, T.W. and Walther, H. (1999) Laser Spectroscopy and Quantum Optics. Reviews of Modern Physics, 71, S242-S252. https://doi.org/10.1103/RevModPhys.71.S242

[21] Rempe, G., Walther, H. and Klein, N. (1987) Observation of Quantum Collapse and Revival in a One-Atom Maser. Physical Review Letters, 58, 353-356. https://doi.org/10.1103/PhysRevLett.58.353

[22] Buck, B. and Sukumar, C.V. (1981) Exactly Soluble Model of Atom-Phonon Coupling Showing Periodic Decay and Revival. Physics Letters A, 81, 132-135. https://doi.org/10.1016/0375-9601(81)90042-6

[23] Sukumar, C.V. and Buck, B. (1981) Multi-Phonon Generalisation of the JaynesCummings Model. Physics Letters A, 83, 211-213. https://doi.org/10.1016/0375-9601(81)90825-2

[24] Yurke, B. and Stoler, D. (1986) Generating Quantum Mechanical Superpositions of 
Macroscopically Distinguishable States via Amplitude Dispersion. Physical Review Letters, 57, 13-16. https://doi.org/10.1103/PhysRevLett.57.13

[25] Gora, P. and Jedrzejek, C. (1992) Nonlinear Jaynes-Cummings Model. Physical Review $A, 45,6816$. https://doi.org/10.1103/PhysRevA.45.6816

[26] Buzek, V. and Jex, I. (1990) Dynamics of a Two-Level Atom in a Kerr-Like Medium. Optics Communications, 78, 425-435. https://doi.org/10.1016/0030-4018(90)90340-Y

[27] Obada, A.-S.F., Eied, A.A. and Al-Kader Abd, G.M. (2008) Treatment of the Emission and Absorption Spectra for a $\Lambda$-Type Three-Level Atom Driven by a Single-Mode Field with Nonlinearities. Laser Physics Letters, 18, 1164-1175. https://doi.org/10.1088/0953-4075/39/7/001

[28] Abdalla, M.S., Krepelka, J. and Perina, J.J. (2006) Effect of Kerr-Like Medium on a Two-Level Atom in Interaction with Bimodal Oscillators. Journal of Physics B: Atomic, Molecular and Optical Physics, 39, 1563-1577. https://doi.org/10.1088/0953-4075/39/7/001

[29] Lai, Y.Z., Li, W.D. and Liang, J.Q. (1999) Adiabatic Transfer of Atomic Level-Occupation Probability Induced by Kerr-Like Medium. Optics Communications, 160, 240-244. https://doi.org/10.1080/09500340108240888

[30] Berlin, G. and Aliaga, J.J. (2001) Quantum Dynamical Properties of a Two-Photon Non Linear Jaynes-Cummings Model. Journal of Modern Optics, 48, 1819-1829. https://doi.org/10.1080/09500340108240888

[31] Schlicher, R.R. (1989) Jaynes-Cummings Model with Atomic Motion. Optics Communications, 70, 97-102. https://doi.org/10.1016/0030-4018(89)90276-9

[32] Liu, J.-R. and Wang, Y.-Z. (1996) Motion-Quantized Jaynes-Cummings Models with an Arbitrary Intensity-Dependent Medium. Physical Review A, 54, 2326. https://doi.org/10.1103/PhysRevA.54.2326

[33] Liu, J.-R. and Wang, Y.-Z. (1996) Velocity-Selective Population and Quantum Collapse-Revival Phenomena of the Atomic Motion for a Motion-Quantized Raman-Coupled Jaynes-Cummings Model. Physical Review A, 54, 2444-2450. https://doi.org/10.1103/PhysRevA.54.2444

[34] Abdel-Wahab, N.H., Amin, M.E. and Mourad, M.F. (2002) Influence of the Stark Shift and the Detuning Parameters on the Entanglement Degree in a Two-Mode Coupling System. Journal of the Physical Society of Japan, 71, 2129-2132. https://doi.org/10.1143/JPSJ.71.2129

[35] Zait, R.A. and Abdel-Wahab, N.H. (2002) Nonresonant Interaction between a Three-Level Atom with a Momentum Eigenstate and a One-Mode Cavity Field in a Kerr-Like Medium. Journal of Physics B, 35, 3701-3712. https://doi.org/10.1088/0953-4075/35/17/307

[36] Zait, R.A. and Abdel-Wahab, N.H. (2002) Influence of Detuning and Kerr-Like Medium on the Interaction of a Three-Level Atom with Squeezed Two-Mode Cavity Field. Physica Scripta, 66, 452. https://doi.org/10.1238/Physica.Regular.066a00425

[37] Abdel-Wahab, N.H. (2005) $N$-Level Atom in a Momentum Eigenstate Interacting with an $(N-1)$ Mode Cavity Field. Physica Scripta, 71, 132-135. https://doi.org/10.1238/Physica.Regular.071a00132

[38] Abdel-Wahab, N.H., Amin, M.E. and Taha, M. (2006) One-Mode Interaction with a Four-Level Atom in a Momentum Eigenstate. Fizika A, 2, 113-124.

[39] Abdel-Wahab, N.H. (2008) A Moving Four-Level N-Type Atom Interacting with 
Cavity Fields. Journal of Physics B: Atomic Molecular and Optical Physics, 41, Article ID: 105502. https://doi.org/10.1088/0953-4075/41/10/105502

[40] Fan, A. and Wang, Z.-W. (1994) Phase, Coherence Properties and the Numerical Analysis of the Field in the Nonresonant Jaynes: Cummings Model. Physical Review $A, 49,1509$. https://doi.org/10.1103/PhysRevA.49.1509

[41] Haken, H. and Wolf, H.C. (2004) Atom- und Quantenphysik, Einfuhrung in Die Experimentellen und Theoretischen Grundlagen. 8th Edition, Springer, Berlin.

[42] Alsing, P., Guo, D.-S. and Carmichael, H.J. (1992) Dynamic Stark Effect for the Jaynes-Cummings System. Physical Review A, 45, 5135-5143. https://doi.org/10.1103/PhysRevA.45.5135

[43] Wallraff, A., Schuster, D.I., Blais, A., Frunzio, L., Huang, R.-S., Majer, J., Kumar, S., Girvin, S.M. and Schoelkopf, R.J. (2004) Strong Coupling of a Single Photon to a Superconducting Qubit Using Circuit Quantum Electrodynamics. Nature, 431, 162-167. https://doi.org/10.1038/nature02851

[44] Alsing, P. and Zubairy, M.S. (1987) Collapse and Revivals in a Two-Photon Absorption Process. Journal of the Optical Society of America B, 4, 177-184. https://doi.org/10.1364/JOSAB.4.000177

[45] Swain, S. (1994) Systematic Method for Deriving Effective Hamiltonians. Physical Review A, 49, 2816. https://doi.org/10.1103/PhysRevA.49.2816

[46] Brune, M., Raimond, J.M. and Haroche, S. (1987) Theory of the Rydberg-Atom Two-Photon Micromaser. Physical Review A, 35, 154-163. https://doi.org/10.1103/PhysRevA.35.154

[47] Agarwal, G.S. and Puri, R.R. (1986) Exact Quantum-Electrodynamics Results for Scattering, Emission, and Absorption from a Rydberg Atom in a Cavity with Arbitrary Q. Physical Review A, 33, 1757-1764. https://doi.org/10.1103/PhysRevA.33.1757

[48] Obada, A.S.F., Ahmed, M.M.A., Khalil, E.M. and Ali, S.I. (2013) Entangled Two Two-Level Atoms Interacting with a Cavity Field in the Presence of the Stark Shift Terms. Optics Communications, 287, 215-223. https://doi.org/10.1016/j.optcom.2012.08.091

[49] Jamal Anwar, S., Ramzan, M. and Khan, K. (2017) Dynamics of Entanglement and Quantum Fisher Information for $N$-Level Atomic System under Intrinsic Decoherence. Quantum Information Processing, 16, 142. https://doi.org/10.1007/s11128-017-1589-8

[50] Berrada, K., Abdel-Khalek, S. and Obada, A.S.F. (2012) Quantum Fisher Information for a Qubit System Placed Inside a Dissipative Cavity. Physics Letters A, 376, 1412-1416. https://doi.org/10.1016/j.physleta.2012.03.023

[51] Berrada, K., Abdel-Khalek, S. and Raymond Ooi, C.H. (2012) Quantum Metrology with Entangled Spin-Coherent States of Two Modes. Physical Review A, 86, Article ID: 033823. https://doi.org/10.1103/PhysRevA.86.033823

[52] Wootters,W.K. (2001) Entanglement of Formation and Concurrence. Quantum Information and Computation, 1, 27-44.

[53] Lu, X., Wang, X. and Sun, C.P. (2010) Quantum Fisher Information Flow and Non-Markovian Processes of Open Systems. Physical Review A, 82, Article ID: 042103. https://doi.org/10.1103/PhysRevA.82.042103

[54] Barndorff-Nielsen, O.E., Gill, R.D. and Jupp, P.E. (2003) On Quantum Statistical Inference. Journal of the Royal Statistical Society Series B, 65, 775-816.

https://doi.org/10.1111/1467-9868.00415 
[55] Abdel-Khalek, S. (2011) Dynamics of a Moving Five-Level Atom Interacting with Cavity Fields. Journal of Russian Laser Research, 32, 86-93.

https://doi.org/10.1007/s10946-011-9192-4

[56] Abdel-Khalek, S. (2013) Quantum Fisher Information for Moving Three-Level Atom. Quantum Information Processing, 12, 3761-3769.

https://doi.org/10.1007/s11128-013-0622-9

[57] Enaki, N.A. and Ciobanu, N. (2008) Quantum Trapping Conditions for Three-Level Atom Flying through Bimodal Cavity Field. Journal of Modern Optics, 55, 589-598. https://doi.org/10.1080/09500340701721868 